\title{
The Art and Science of Polymer Brushes: Recent Developments in Patterning and Characterization Approaches
}

\author{
Guido Panzarasa*
}

\begin{abstract}
Polymer brushes are dense arrays of macromolecular chains tethered by one end at a surface. They are at the cutting edge of polymer nanotechnology since the dawn of controlled surface-initiated polymerization techniques unlocked new prospects for the synthesis of polymer brushes with tailorable properties. More recently, thanks to the growing interest in the use of brushes for the generation of functional surfaces, the need for advanced patterning and characterization approaches rapidly increased. Meeting these needs requires the contribution of experts from different disciplines: polymer chemistry, surface science, electrochemistry and particle physics. The focus of this review is to highlight recent developments in the field of polymer brushes, specifically the application of photocatalytic lithography as a versatile patterning strategy, the study of grafted-from polymer brushes by electrochemical methods and, most importantly, the introduction of positron annihilation spectroscopy as a powerful technique for the investigation of the structure of polymer brushes and of their composites with nanoparticles.
\end{abstract}

Keywords: Electrochemistry · Photocatalytic lithography · Polymer brushes · Positron annihilation spectroscopy

\section{Introduction}

"Polymer 'brushes' are long-chain polymer molecules attached by one end to a surface or interface by some means, with a density of attachment points high enough so that the chains are obliged to stretch away from the interface [...] like the bristles in a brush, hence the name" [1] The history of polymer brush research is long and successful: for decades, starting from the mid 1900s, this field has been at the forefront of research in the polymer community. Initially, the greatest advances were in the theoretical domain, contributing to lay the physico-mathematical foundation of models which are still valid today. ${ }^{[2]}$

Experimental research, however, could not keep pace with theoretical advances until controlled radical polymerization

${ }^{*}$ Correspondence: Dr. G. Panzarasa

Università del Piemonte Orientale 'Amedeo Avogadro' Department of Science and Technological Innovation viale T. Michel 11, 15100 Alessandria, Italy

Current address: Empa Materials Science and

Technology

Materials Meet Life Department

Laboratory for Biomimetic Membranes and Textiles

Lerchenfeldstrasse 5, CH-9014 St. Gallen

E-mail: gp4779@gmail.com techniques were introduced. ${ }^{[3]}$ Nitroxidemediated polymerization (NMP), ${ }^{[4]}$ reversible addition-fragmentation transfer polymerization (RAFT) ${ }^{[5]}$ and atom transfer radical polymerization (ATRP) ${ }^{[6]}$ represented a real breakthrough in polymer synthesis, opening new possibilities for the design and tailoring of macromolecular structures to the broadest chemical community. From bulk synthesis to surfaceinitiated polymerization the step was short and research on polymer brushes received an incredible impulse from such userfriendly, versatile and flexible approaches to grow polymer chains directly from surfaces in a controlled, programmable fashion. ${ }^{[7]}$

The first step in the fabrication of polymer brushes is to deposit a self-assembled monolayer (SAM) of a suitable initiator on the substrate of choice. SAMs are thin films which can form spontaneously from chemisorption of organic molecules on solid surfaces thanks to specific interactions between functional groups on the molecule and on the surface. Their relevance for the modification of surface properties is well known: potential applications include wetting, friction, adhesion, sensing and lithography:[8] in this context, the ability to pattern SAMs of initiators is an important key for advanced applications of polymer brushes. The second step involves the amplification of the initiator SAM into polymer brushes by growing polymer chains by surface-initiated polymerization. Eventually, the brushes can be post-functionalized by performing different reactions and/or decorated with nanoparticles. ${ }^{[9]}$

Polymer brushes, once only a useful model for polymer science problems, rapidly became a fruitful playground for highly interdisciplinary research. This led to countless applications ranging from biomedicine to electronics, from controlled drug release to tribology, from sensors and actuators to smart surfaces, to name a few. ${ }^{[10]}$ However, their very nature poses challenges for characterization and new analytical approaches are required. Here, some of the recent advances in patterning and characterization of polymer brushes are reviewed.

\section{Patterning of Polymer Brushes by Direct and Remote Photocatalytic Lithography}

The last decades have witnessed a dramatic progress in micro- and nanofabrication, with an increasing focus towards hybrid techniques. Needless to say, the field of polymer brushes has benefited from these new developments in current research. ${ }^{[11]}$ Obtaining spatially resolved patterns of polymer brushes is of critical importance for both fundamental studies and for the development of functional surfaces, (bio)sensors and actuators. ${ }^{[12]}$

According to the process by which polymer brushes are patterned, it is possible to distinguish between direct and indirect patterning strategies. ${ }^{[13]}$ Direct patterning is a 'top-down' approach, in which 
chains of pre-formed polymer brushes are selectively degraded by means of locally applied mechanical force (e.g. shaving), by irradiation with light or with particle beams (breaking of chemical bonds). On the other hand, indirect patterning is a 'bottom-up' approach, in which patterns of surface-grafted initiators are first prepared and subsequently amplified into polymer brushes by means of surface-initiated polymerization techniques (Fig. 1A). Thanks to its versatility, this latter approach is the most frequently used one. High-resolution patterns of initiators can be generated by strategies based on irradiation (e.g. photoand interference lithography, electron- and ion-beam lithography), ${ }^{[14]}$ mechanical contact (e.g. scanning probe lithography, soft lithography, microcontact printing, nanoimprinting lithography), ${ }^{[15]}$ and on surface forces (colloidal lithography). ${ }^{[16]}$

Ideally, these lithographic techniques for the patterning of polymer brushes should be reliable, applicable on a waferscale and, at the same time, provide access to high resolution micro- and nanometric features. Among these different patterning methods, photolithography allows an entire pattern to be transferred from a photomask to a SAM with a single exposure with good reproducibility, making it a convenient approach. However, conventional photolithography involves the use of polymeric resists, which can be difficult to remove, while direct UV lithography requires powerful and potentially dangerous light sources. A demand for affordable and facile approaches to expand the existing technologies still constitutes a challenge, which could be successfully overcome by means of photocatalytic lithography.

The absorption of photons of sufficient energy by a photoactive semiconductor can provoke photocatalytic reactions at its surface, e.g. water splitting or the degradation of organic compounds. Anatase titanium dioxide is one of the most popular and studied photoactive semiconductors, ${ }^{[17]}$ but other photocatalytic oxides such as $\mathrm{WO}_{3}$ and $\mathrm{ZnO}$ could be used as well. ${ }^{[18]}$ Upon irradiation with UV light, electron-hole pairs are generated in the semiconductor, which subsequently produce highly reactive oxygen species (e.g. hydroxyl radical, hydrogen peroxide) from adsorbed oxygen and water molecules. These reactive species are used to decompose organic molecules at the surface, ${ }^{[19]}$ leading to the patterning of $e$.g. polymerization initiators.

Photocatalytic lithography can be carried out in a 'direct' or in a 'remote' fashion. (Fig. 1B). In the direct photocatalytic lithography approach, the pattern is obtained directly on a titania surface, while in the remote approach a transparent titania-coated quartz or glass substrate is positioned at a distance of up to $100 \mu \mathrm{m}$ from the surface to be patterned. Remote

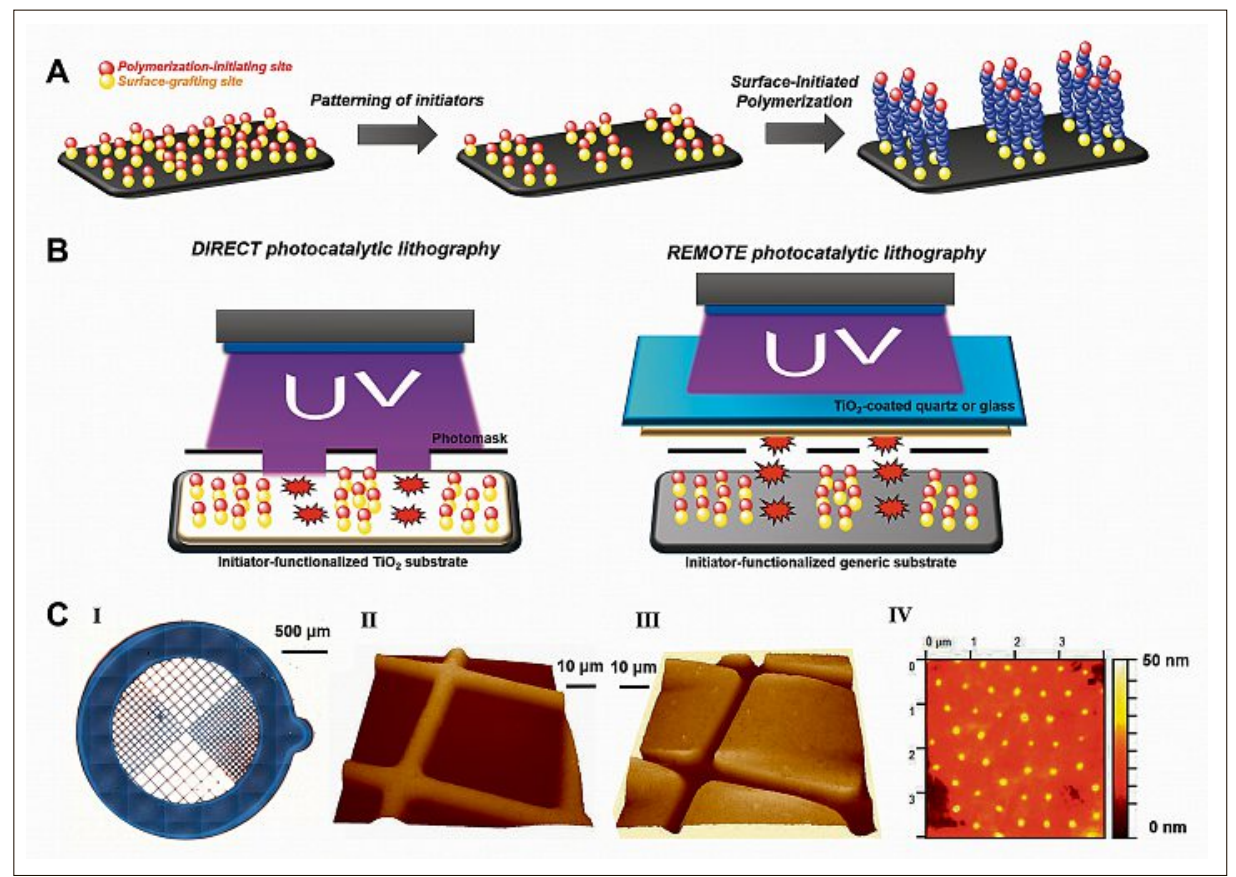

Fig. 1. A) Synthetic strategy of a bottom-up approach for the patterning of polymer brushes. B) Schematic representation of direct and remote photocatalytic lithography, in which the red forms symbolize reactive oxygen species. C) Polymer brush patterns obtained by remote (I, IV) and direct (II, III) photocatalytic lithography. (I) optical microscopy image of a positive polymer brush pattern (adapted from ref. [22a]); (II, III) atomic force microscopy (AFM) images of a positive and a negative polymer brush pattern (adapted from ref. [23]); (IV) AFM image of a polymer brush nanopattern obtained by colloidal lithography coupled with remote photocatalysis (adapted from ref. [23]).

photocatalytic lithography exploits the ability of some reactive oxygen species, especially hydrogen peroxide, to diffuse from the surface on which they are generated to the substrate, where they can exert their oxidative potential.[20] Compared to direct photocatalytic lithography, the remote approach presents many advantages: it is substrate-independent, the titaniacoated substrate can be reused for many cycles, the resolution is comparable and the process time for patterning is relatively short. Photocatalytic lithography has already proven to be useful for the development of superhydrophilic/phobic patterns, in offset printing, for the development of (bio)sensors and for the generation of metal particles arrays. ${ }^{[21]}$ Recently the potential of this technique for the patterning of polymer brushes was demonstrated by our group. ${ }^{[22]}$ Some examples of the obtained structures are shown in Fig. 1C. Positive as well as negative replicas of a photomask can be obtained: in the latter case, a sacrificial monolayer is patterned first and empty spaces are back-filled with a suitable initiator. The combination of remote photocatalysis and colloidal lithography opens up the possibility to reach nanometer-sized complex patterns by using a SAM of particles on an initiator-grafted surface. ${ }^{[23]}$

Photocatalytic lithography can be made accessible to a broader community since it requires simpler instrumentation, is more user-friendly and faster than conventional photolithography. Moreover, low-power, $365 \mathrm{~nm}$ UV light sources can be used and the use of polymeric photoresists is avoided.

\section{Grafted-from Polymer Brushes Allowing Control of the Electrochemical Properties of Silicon Substrates}

Silicon is the workhorse of micro- and nanofabrication: its technological importance is steadily increasing as it finds countless applications in the contemporary enabling technologies. However, such a versatile material has also limitations, such as the oxide that naturally covers its surface: useful in conventional structuring techniques, it impairs dramatically the electrochemical properties of silicon due to lack of affinity towards conventional redox probes and its barrier properties towards electron transfer. ${ }^{[24]}$ Silicon, unless highly doped, displays very poor properties as a working electrode in conventional electrochemical cells, which is a serious limitation for the development of sensors, lab-on-chip and microfluidic devices that would otherwise benefit from such an affordable, versatile and easily scalable substrate. ${ }^{[24]}$ 
Silicon wafer is also the substrate of choice to perform grafting-from of polymer brushes and it was recently discovered that polymer brushes made from hydrophilic and functional monomers can efficiently control its electrochemical behavior.[25]

Homopolymer and random co-polymer brushes of poly(2-hydroxyethyl methacrylate) (PHEMA) and poly(2-aminoethyl methacrylate) (PAMA), as well as brushes of poly(methacrylic acid) (PMAA), were grown from initiator-functionalized silicon wafers by means of SI-ATRP (Fig. 2A). Electrochemical impedance spectroscopy (EIS) and cyclic voltammetry (CV) were then used to investigate the electrochemical behavior of the brush-modified silicon electrodes. For the PHEMA, PAMA and PHEMA-PAMA random copolymer brushes, EIS clearly showed, for the brushmodified electrodes, a dramatic reduction in the resistance as well as a marked inter-

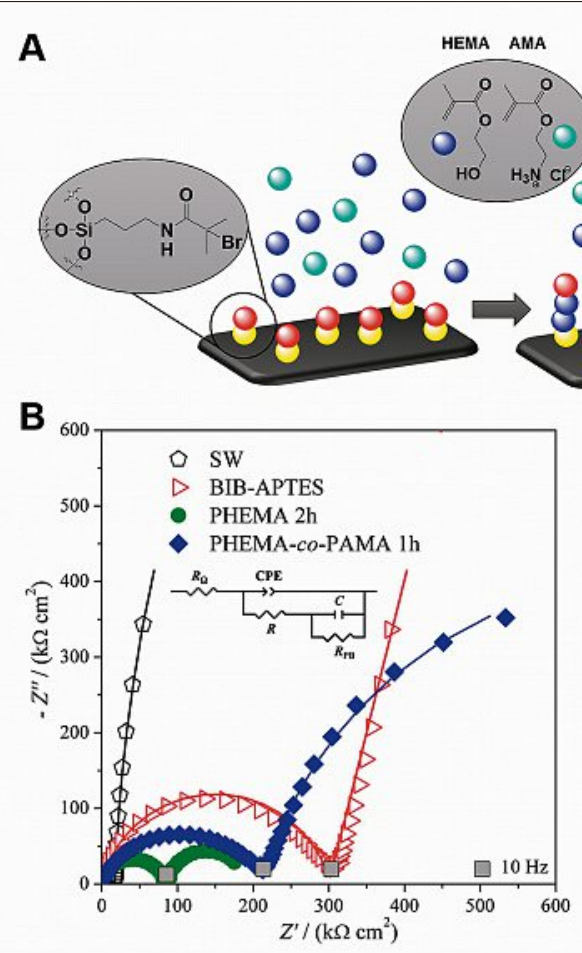

C

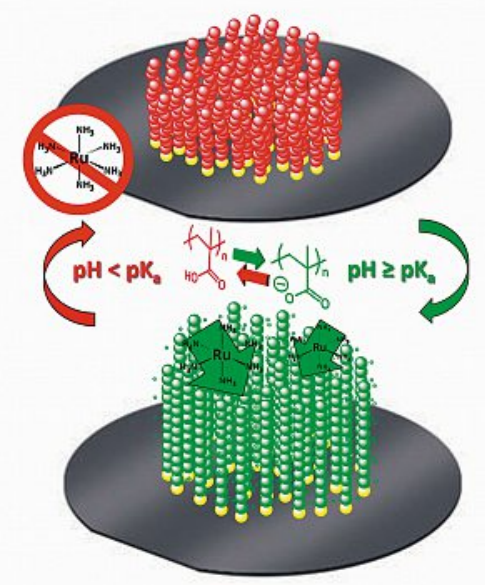

$100^{0}$

(3)
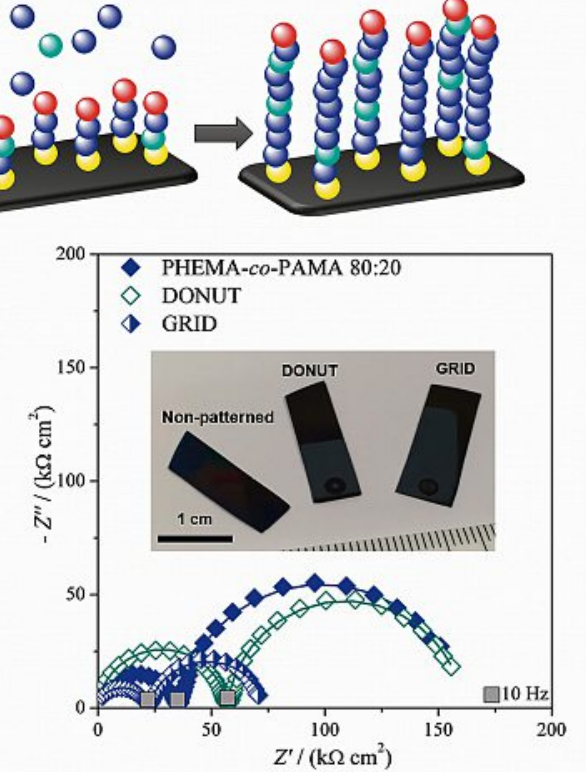

D

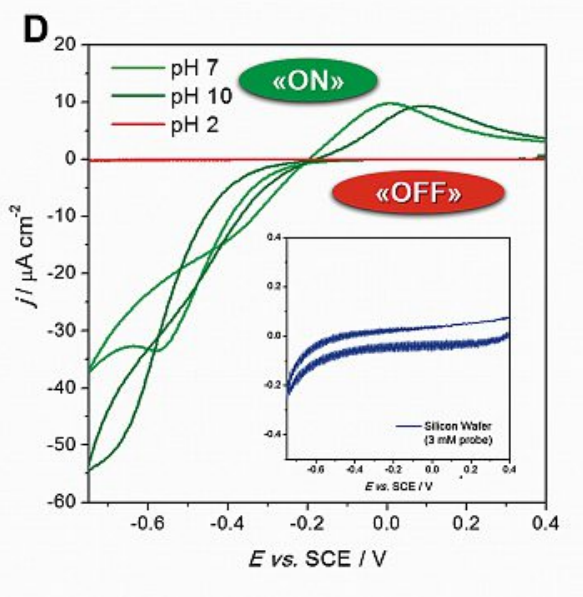

Fig. 2. A) Synthetic strategy for the grafting of PHEMA-PAMA random copolymer brushes from silicon substrates (adapted from ref. [25a]). B) Left: EIS spectra for pristine silicon wafer (SW), silicon wafer modified with the ATRP-initiator (BIB-APTES), silicon wafer modified with PHEMA brushes (PHEMA 2h) and with PHEMA-PAMA random copolymers (PHEMA-PAMA 80:20 1h). The inset shows the resulting electrical circuit to model the brush-modified silicon electrode. Right: EIS spectra for non-patterned (PHEMA-PAMA 80:20), macropatterned (DONUT) and micropatterned (GRID) brush-modified silicon substrate. The inset shows a picture of the electrodes. The experiments were carried out at $-0.1 \mathrm{~V},+0.1 \mathrm{~V}$ and $+0.25 \mathrm{~V}(\mathrm{SCE})$ in the presence of $3 \mathrm{mM} \mathrm{K}_{4} \mathrm{Fe}(\mathrm{CN})_{6}$ using $0.1 \mathrm{M} \mathrm{KCl}$ as the supporting electrolyte (adapted from ref. [25a]). C) Schematic representation of the $\mathrm{pH}$-responsive behavior of PMAA brushes governing their interaction with the redox probe ruthenium(II) hexamine (adapted from ref. [25b]). D) CV spectra of PMAA brushes at different $\mathrm{pH}$ values. The inset shows the CV of pristine silicon wafer obtained in the same conditions. The experiments were performed by scanning the potential between $-0.75 \mathrm{~V}$ and $+0.4 \mathrm{~V}$ (SCE) with a scan rate of $0.1 \mathrm{~V} \mathrm{~s}^{-1}$ (adapted from ref. [25b]).

action with the redox probe, which were absent for both the pristine and for the initiator-modified substrates. Moreover, micropatterning of the brushes by means of remote photocatalytic lithography allowed to achieve a greater control of the electrochemical response (Fig. 2B), most probably due to the increased surface area. ${ }^{[25 a]}$

However, the most interesting feature was discovered by analyzing the electrochemical behavior of polyelectrolyte brushes (Fig. 2C,D). A polyelectrolyte is a polymer that carries ionic groups on its repeat units. Poly(methacrylic acid) is a polyelectrolyte which carries carboxylic groups with a mean $\mathrm{pK}_{\mathrm{a}}$ of 6.5 . For $\mathrm{pH}<$ $\mathrm{pK}$, the carboxylic groups are protonated and electrically neutral while for $\mathrm{pH}>\mathrm{pK}_{\mathrm{a}}$ the majority of them are deprotonated and negatively charged. This well-known $\mathrm{pH}-$ controlled mechanism has already been exploited for different applications ${ }^{[26]}$ and more recently was demonstrated to allow the switching of the electrochemical properties of silicon. ${ }^{25 b]}$ As shown by cyclic voltammetry (Fig. 2D), no electrochemical signal could be detected when the brushes were protonated $(\mathrm{pH}=2)$, meaning that no electron transfer was taking place between the electrode surface and the redox probe (i.e. a redox-active molecule with stable and well-known electron transfer properties, such as ferrocyanide and ruthenium hexaamine). Conversely, in neutral $(\mathrm{pH}=$ 7) and basic $(\mathrm{pH}=10)$ media, i.e. for partially and fully deprotonated brushes, the oxidation and reduction peaks of the redox probe were clearly visible, demonstrating that electron transfer was actually taking place. To further emphasize the magnitude of the effect, the inset in Fig. 2D shows the $\mathrm{CV}$ of pristine silicon wafer obtained under the same experimental conditions. Another remarkable phenomenon was observed, that is the crossing of the $\mathrm{CV}$ lines at two points: it could be considered as an indication of a memristive behavior, which has been observed only recently for polymer brushes-modified electrodes and could open new areas of application for the development of, for example, hybrid logic gates. ${ }^{[27]}$

\section{Positron Annihilation Spectroscopy: An Enabling Technique for the Study of Polymer Brushes}

Positron annihilation spectroscopy (PAS) is a well-established non-destructive technique for the determination of nanoscale porosity, and generally speaking of free volume, in many different materials (metals, semiconductors, oxides, polymers, colloids).[28] In PAS, positrons $\left(\mathrm{e}^{+}\right)$, the anti-particles of electrons, emitted 


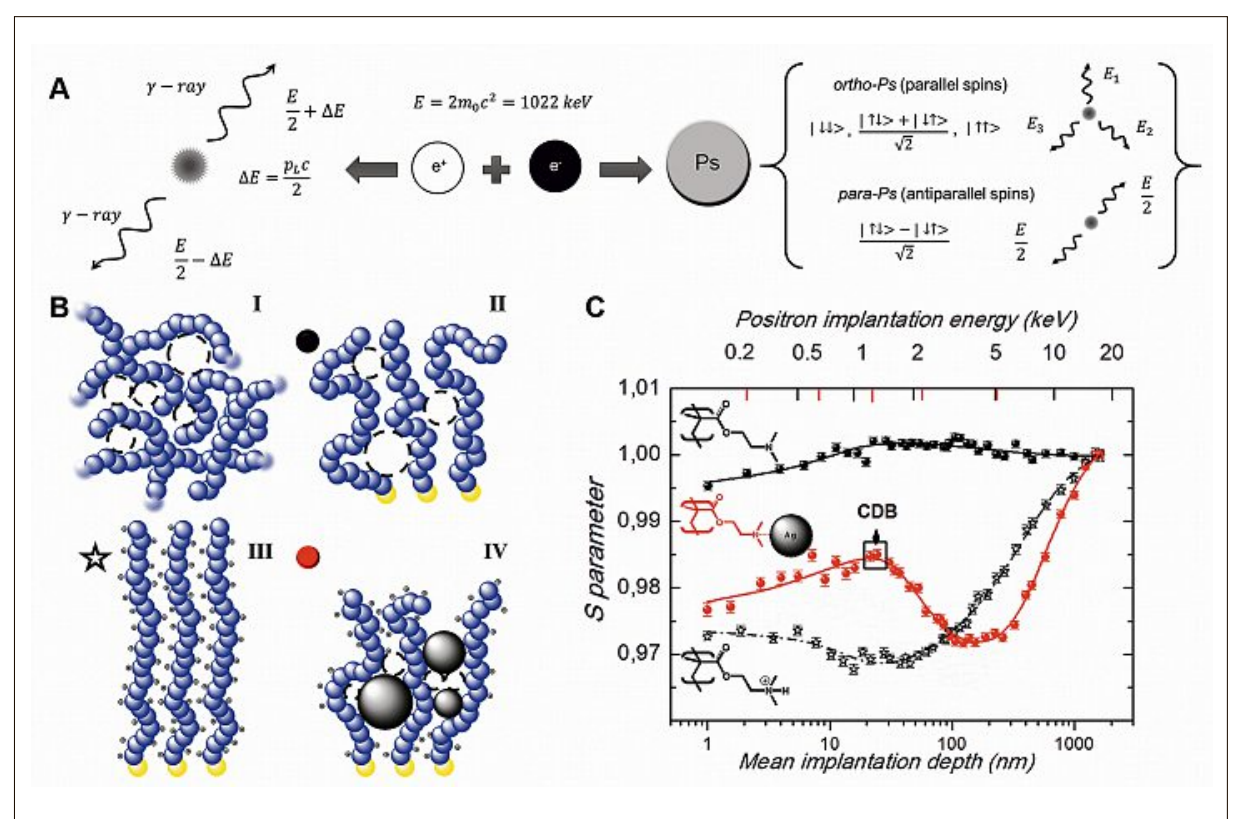

Fig. 3. A) Schematic representation of positron-electron annihilation, positronium formation and annihilation (adapted from ref. [33]). B) Schematic representations of: (I) chains and free volume holes in a bulk polymer sample; (II) chains arrangement in a collapsed polymer brush; (III) chains arrangement in a highly-stretched, protonated polymer brush (the negative counterions are omitted for simplicity); (IV) chains arrangement in a negatively-charged metal particles-decorated protonated brush. Polymer chains are represented as blue pearl-strings, free volume holes as hollow circles, the yellow pearls are the grafting sites and the grey pearls represent metal particles. The black dot, hollow star and red dot symbols mean that these structures represent also the systems analyzed in (C). C) Graph showing the evolution of the S-parameter as a function of the positron implantation energy and the mean implantation depth. The mean brush thickness is around 100 $\mathrm{nm}$ : the rapid increase of signal for higher values originates from positrons implanting in the silicon substrate. Protonation of the brushes was achieved by immersion in dilute nitric acid, decoration with silver nanoparticles was achieved by immersion in an aqueous suspension of pre-made citrate-stabilized, borohydride-reduced silver nanoparticles (adapted from ref. [33]).

from a radioactive source (typically ${ }^{22} \mathrm{Na}$ ) are implanted into the studied material. The interaction between a positron and an electron leads either to immediate annihilation, their mass being entirely converted into energy in the form of gamma-rays $(\gamma$-rays), or can combine temporarily into an exotic atomic form called positronium (Ps) (Fig. 3A). The Ps is similar to an hydrogen atom in which the proton is substituted by the positron, but in contrast to ${ }^{1} \mathrm{H}$ it annihilates rapidly into $\gamma$-rays. ${ }^{[29]}$ For polymers, the regions of low electron density, where Ps can be formed, correspond to free volume cavities with a radius of $0.2-0.6 \mathrm{~nm}$. Two spin states are possible, namely para-positronium (p-Ps) and orthopositronium (o-Ps), with the latter having a longer lifetime of about $142 \mathrm{~ns}$ in vacuum. However, o-Ps localized in a free volume hole can annihilate with an electron from the atoms of the cavity walls in a significantly shorter time: this process is called pick-off annihilation and is the main pathway of Ps annihilation in polymers. [30]

When a positron and an electron annihilate, the total energy released is 1.022 $\mathrm{MeV}$, which accounts for the combined masses at rest of the two particles. As the total energy of the system has to be conserved, the annihilation process gener- ates, in most cases, two photons of equal energy (511 keV each) which are emitted at almost $180^{\circ}$ and their intensity measured. The ratio of the peak counts to total counts in a curve of detected gamma radiation represents low-momentum positrons and corresponds to positron annihilation with the valence electrons. This is called the S-parameter and is sensitive to open volume defects. In bulk solids, its increase indicates the presence of vacancy defects. Moreover, it is important to note that because of the finite momentum of the electron-positron pair, the annihilation energy of $511 \mathrm{keV}$ gets Doppler-shifted by an amount $\Delta \mathrm{E}$. Since numerous annihilation events are measured to give the complete Doppler spectrum, the energy line is broadened due to the individual Doppler shifts along the annihilation direction. Coincidence Doppler broadening (CDB) spectroscopy gives information on the electron momentum distribution in the sample and ultimately, gives insight on the elemental composition of the annihilation cavity walls. ${ }^{[31]}$ Another important parameter is the lifetime of positron and positronium, which is the inverse of the rate of annihilation and is inversely proportional to the electron density at the annihilation site. For these reasons, the lifetime is re- lated to the size of the free volume while the signal intensity is related to the number concentration of free volume. Notably, the chemistry of functional groups present in the polymer can also have a strong influence on the lifetime as well as on the $\gamma$-rays emission intensity. [32]

Positron annihilation spectroscopy is an established technique in polymer science, but its application for the analysis of polymer brushes has been reported only recently. ${ }^{[33]}$ By means of a variable energy positron beam (VEPAS), brushes made of poly(dimethylaminoethyl methacrylate) (PDMAEMA) were analyzed in three different states: pristine, protonated and after incorporation of silver nanoparticles (Fig. 3B). The use of such a variable-energy positron beam is necessary to control the implantation depth of positron in less than micrometer-thick samples and allows to achieve a spatial description of the samples as well. As shown in Fig. 3C, the $\mathrm{S}$-parameter evolution is dramatically different for the three analyzed brushes. For the as-made brushes, the implanted positron forms p-positronium which remains confined in free volume cavities, explaining the absence of features. For the protonated brushes, on the other hand, a significant drop in p-positronium occurs, most probably because of electrostatic repulsion and the fact that the highly stretched chains are more ordered compared to the collapsed state, reducing the overall free volume. In the case of brushes decorated with silver nanoparticles, a strong chemical modification of the annihilation sites is apparent (also from the CDB, which gives the fingerprint of silver) and the $\mathrm{p}$ positronium yield is increased compared to the protonated brushes, testifying the generation of cavities able to form $\mathrm{p}$ positronium, most probably due to the disorder introduced by the particles. From data analysis and fitting with dedicated algorithms (VEPFIT), ${ }^{[34]}$ values such as the density and the particles distribution profile of the analyzed samples could be extracted without damaging or destroying the sample, as in the case of electron microscopy. Comparison with data obtained with more conventional techniques such as X-ray reflectivity (XRR) showed excellent agreement for the pristine and protonated brushes, but clearly showed the superiority of PAS for investigating more complex (e.g. nanoparticle-decorated) systems, for which XRR failed to give a proper density estimation.

\section{Conclusions}

Polymer brushes are one of the most promising and challenging fields of polymer-based nanotechnology. In this per- 
spective some of most recent achievements in this area of investigation were reviewed, namely: i) the introduction of photocatalytic lithography as a versatile tool for the patterning of polymer brushes, ii) the demonstration that polymer brushes allow to control the electrochemical properties of silicon opening new fields of application for the development of hybrid logic gates and electrochemical devices, iii) the promises of positron annihilation spectroscopy as a non-destructive technique for the analysis of polymer brushes and of their composites with nanoparticles, which will set a milestone for the structural analysis of such complex and fascinating systems.

\section{Acknowledgments}

Many people must be acknowledged for the results presented in this review. First, my advisor Dr. Katia Sparnacci, for her continuous support during my $\mathrm{PhD}$ project. Then, my colleagues: Dr. Guido Soliveri, Prof. Silvia Ardizzone, Dr. Valentina Pifferi, Prof. Luigi Falciola (Università degli Studi di Milano), Prof. Giovanni Consolati, Dr. Stefano Aghion, Prof. Rafael Ferragut (Politecnico di Milano), Dr. Matthias Dübner (ETH Zürich) and Dr. Celestino Padeste (PSI Villigen) for all the work we performed together. My greatest gratitude goes to the researchers of the Eni Donegani Research Institute for Renewable Energies and the Environment (Novara): Dr. Gianluigi Marra, Dr. Laura Meda, Dr. Alberto Savoini and Dr. Mario Salvalaggio. Last but not least, I would like to thank Dr. Alina Osypova (Empa St. Gallen) for reviewing the manuscript.

Received: April 13, 2017

[1] S. T. Milner, Science 1991, 251, 905.

[2] a) S. T. Milner, T. A. Witten, M. E. Cates, Macromolecules 1988, 21, 2610; b) P.-G. de Gennes, Rep. Prog. Phys. 1969, 32, 187; c) P.G. de Gennes, Adv. Colloid Interf. Sci. 1987, 27, 189; d) A. M. Skvortsov, I. V. Pavlushkov, A. A. Gorbunov, Ye. B. Zhulina, O. V. Borisov, V. A. Pryamitsyn, Polym. Sci. USSR 1988, 30, 1706; e) S. Alexander, J. de Phys. 1977, 38, 983; f) P. Pincus, Macromolecules 1991, 24, 2912.

[3] W. A. Braunecker, K. Matyjaszewski, Prog. Polym. Sci. 2007, 32, 93.

[4] R. H. Grubbs, Polym. Rev. 2011, 51, 104.
[5] G. Moad, E. Rizzardo, S. H. Thang, Polymer 2008, 49, 1079.

[6] K. Matyjaszewski, Macromolecules 2012, 45, 4015 .

[7] a) K. Matyjaszewski, Prog. Polym. Sci. 2005, 30, 858; b) K. Matyjaszewski, H. Dong, W. Jakubowski, J. Pietrasik, A. Kusumo, Langmuir 2007, 23, 4528; c) B. Li, B. Yu, Q. Ye, F. Zhou, Acc. Chem. Res. 2015, 48, 229.

[8] a) A. Ulman, Chem. Rev. 1996, 96, 1533; b) J. C. Love, L. A. Estroff, J. K. Kriebel, R. G. Nuzzo, G. M. Whitesides, Chem. Rev. 2005, 105, 1103; c) T. Schmaltz, G. Sforazzini, T. Reichert, H. Frauenrath, Adv. Mater. 2017, DOI: 10.1002/adma.201605286.

[9] a) M. Dübner, M.-E. Naoum, N.D. Spencer, C. Padeste, ACS Omega 2017, 2, 455; b) M. Dübner, T. N. Gevrek, A. Sanyal, N. D. Spencer, C. Padeste, ACS Applied Mater. Interfaces 2015, 7, 11337; c) M. Dübner, N. D. Spencer, C. Padeste, Langmuir, 2014, 30, 14971; d) E. S. Dehghani, N. D. Spencer, S. N. Ramakrishna, E. M. Benetti, Langmuir 2016, 32, 10317.

[10] a) R. Barbey, L. Lavanant, D. Paripovic, N. Schüwer, C. Sugnaux, S. Tugulu, H.-A. Klok, Chem. Rev. 2009, 109, 5437; b) J. O. Zoppe, N. C. Ataman, P. Mocny, J. Wang, J. Moraes, H.-A. Klok, Chem. Rev. 2017, 117, 1105; c) M. Krishnamoorthy, S. Hakobyan, M. Ramstedt, J. E. Gautrot, Chem. Rev. 2014, 114, 10976; d) N. Ayres, Polym. Chem. 2010, 1, 769; e) J. J. Keating, J. Imbrogno, G. Belfort, ACS Appl. Mater. Interf. 2016, 8, 28383; f) T. Kreer, Soft Matter 2016, 12, 3479; g) M. Dübner, V. J. Cadarso, T. N. Gevrek, A. Sanyal , N. D. Spencer, C. Padeste, ACS Applied Mater. Interfaces 2017, 9, 9245.

[11] a) S. R. Quake, A. Scherer, Science 2000, 290, 5496; b) E. Menard, M. A. Meitl, Y. Sun, J.-U. Park, D. J.-L. Shir, Y.-S. Nam, S. Jeon, J. A. Rogers, Chem. Rev. 2007, 107, 1117; c) A. del Campo, E. Arzt, Chem. Rev. 2008, 108, 911.

[12] T. Chen, I. Amin, R. Jordan, Chem. Soc. Rev 2012, 41, 3280.

[13] A. Biswas, I. S. Bayer, A. S. Biris, T. Wang, E. Dervishi, F. Faupeld, Adv. Colloid Interf. Sci. 2012, 170, 2.

[14] a) J.-K. Chen, Z-Y, Chen, H.C. Lin, P.-D. Hong and F.-C. Chang, ACS Appl. Mater. Interfaces 2009, 1, 1525; b) M. Dübner, T.N. Gevrek, A. Sanyal, N. D. Spencer, C. Padeste, ACS Appl. Mater. Interfaces 2015, 7, 11337; c) K. Gajos, V. A. Guzenko, M. Dübner, J. Haberko, A. Budkowski, C. Padeste, Langmuir 2016, 32, 10641; d) N. Herzer, S. Hoeppener, U. S. Schubert, Chem. Commun. 2010, 46, 5634.

[15] a) T. Chen, R. Jordan, S. Zauscher, Polymer 2011, 52, 2461; b) M. Kaholek, W. Lee, B. LaMattina, K. C. Caster, S. Zauscher, Nano Lett. 2004, 4, 373.

[16] a) Y. Li, J. Zhang, L. Fang, L. Jiang, W. Liu, T. Wang, L. Cui, H. Sun, B. Yang, J. Mater. Chem. 2012, 22, 25116; b) T. Chen, D. P. Chang, R.
Jordan, S. Zauscher, Beilstein J. Nanotechnol. 2012, 3, 397.

[17] a) X. Chen, S. S. Mao, Chem. Rev. 2007, 107, 2891; b) U. Diebold, Surf. Sci. Rep. 2003, 48, 53.

[18] W. Kubo, T. Tatsuma, J. Mater. Chem. 2005, 15, 3104.

[19] K. Nakata, A. Fujishima, J. Photochem. Photobiol. C: Photochem. Rev. 2012, 13, 169.

[20] a) W. Kubo, T. Tatsuma, A. Fujishima, H Kobayashi, J. Phys. Chem. B 2004, 108, 3005 ; b) T. Tatsuma, W. Kubo, A. Fujishima, Langmuir 2002, 18, 9632; c) T. Tatsuma, S.I. Tachibana, A. Fujishima, J. Phys. Chem. B 2001, 105, 6987.

[21] a) J. P. Lee, M. M. Sung, J. Am. Chem. Soc. 2004, 126, 28; b) W. Kubo, T. Tatsuma, Appl. Surf. Sci. 2005, 243, 125; c) T. Tatsuma, Y. Yoshida, I. Shitanda, H. Notsu, Analyst 2009, 134, 223; d) G. Panzarasa, G. Soliveri, G. Marra, L. Media, A. Savoini, S. Ardizzone, M. Salvalaggio, Nanotechnology 2017, 28, 155302.

[22] a) G. Panzarasa, G. Soliveri, K. Sparnacci, S. Ardizzone, Chem. Commun. 2015, 51, 7313; b) G. Panzarasa, G. Soliveri, S. Ardizzone, K. Sparnacci, Mater. Today. Proc. 2015, 2, 4183.

[23] G. Panzarasa, G. Soliveri, S. Ardizzone, Coll. Surf. A: Physicochem. Eng. Aspects 2016, 506, 833.

[24] S. Xun, X. Song, L. Wang, M. E. Grass, Z. Liu, V. S. Battaglia, G. Liu, J. Electrochem. Soc. 2011, 158, A 1260.

[25] a) G. Panzarasa, G. Soliveri, V. Pifferi, J. Mater. Chem. C 2016, 4, 340; b) G. Panzarasa, M Dübner, V. Pifferi, G. Soliveri, C. Padeste, J. Mater. Chem. C 2016, 4, 6287.

[26] a) M. G. Santonicola, G. W. De Groot, M Memesa, A. Meszynska, G. J. Vancso, Langmuir 2010, 26, 17513; b) Z. Qu, H. Xu, H. Gu, ACS Appl. Mater. Interfaces 2015, 7, 14537.

[27] T. K. Tam, M. Pita, O. Trotsenko, M. Motornov, I. Tokarev, J. Halámek, S. Minko, E. Katz, Langmuir 2010, 26, 4506.

[28] a) R. W. Siegel, Annu. Rev. Mater. Sci. 1980, 10, 393; b) Y. C. Jean, J. D. Van Horn, W.-S. Hung, K.-R. Lee, Macromolecules 2013, 46, 7133.

[29] G. Consolati, R. Ferragut, A. Galarneau, F. Di Renzo, F. Quasso, Chem. Soc. Rev. 2013, 42, 3821.

[30] X. S. Li, M. Boyce, J. Polym. Sci. B: Polym. Phys. 1993, 31, 869.

[31] I. Makkonen, C. Rauch, J.-M. Mäki, F. Tuomisto, Physica B, 2012, 407, 2684.

[32] M.V. Félix, G. Consolati, R. Velázquez, V.M. Castaño, Polymer 2006, 47, 265.

[33] G. Panzarasa, S. Aghion, G. Soliveri, G Consolati, R. Ferragut, Nanotechnology 2016, 27, 02LT03.

[34] A. van Veen, H. Schut, J. de Vries, R. A Hakvoort, M. R. Ijpma, AIP Conf. Proc. 1991, 218,171 . 